\title{
Study of the Scattering-To-Absorption Ratio in Plasmonic Nanoparicles for Photovoltaic Cells and Sensors Applications
}

Nader Daneshfar ( $\nabla$ ndaneshfar@gmail.com )

Razi University https://orcid.org/0000-0002-3764-6847

\section{Research Article}

Keywords: Plasmonic nanoparticle, Scattering, Absorption.

Posted Date: March 9th, 2021

DOl: https://doi.org/10.21203/rs.3.rs-206252/v1

License: (1) (1) This work is licensed under a Creative Commons Attribution 4.0 International License.

Read Full License 


\title{
Study of the scattering-to-absorption ratio in plasmonic nanoparicles for photovoltaic cells and sensors applications
}

\author{
NADER DANESHFaR \\ Department of Physics, Faculty of Science, Razi University, Kermanshah, Iran \\ E-mail: ndaneshfar@gmail.com,ndaneshfar@razi.ac.ir
}

Compiled February 4, 2021

\begin{abstract}
:
In this paper, the scattering-to-absorption ratio that is a measurable factor and defined as the ratio of the scattering cross section to the absorption cross section in plasmonic core-shell nanoparticles with different shapes is investigated. The effect of various plasmonic materials on the scattering-to-absorption ratio is presented that can play a key role in plasmonic solar cell efficiency improvement. In addition to using plasmonic materials as silver and gold, we use poor metals as bismuth because this type of metals are good materials for UV plasmonics. We also use semiconductor copper chloride $(\mathrm{CuCl})$ in our calculations, because this material exhibits a strong exciton resonance, while the interaction between the plasmon resonance in metals and the exciton resonance in $\mathrm{CuCl}$ leads to new plexcitonic modes.
\end{abstract}

Keywords: Plasmonic nanoparticle, Scattering, Absorption.

\section{INTRODUCTION}

The interaction between the electromagnetic field and metal nanoparticles can cause significant optical phenomena, which makes them extraordinary scatterers and absorbers of light. In fact, nanometer-sized metallic particles which support plasmon resonances at optical and near-optical frequencies can manipulate light-matter interactions to induce absorption and scattering through their localized surface plasmon resonances [1]. These unique optical effects make nanoparticles suitable for many practical applications such as photovoltaic cells or solar cells, optical devices, sensing, detection and spectroscopy, biological Imaging and biomedicine [2-5].

The phenomena of absorption and scattering of an electromagnetic wave by particles specially nano-scaled size are numerous and have significant applications in optics, communications and sensors [6]. The optical absorption and scattering behavior of metallic nanoparticles with different shape is a topic of interest. When light is incident on a particle can be absorbed or scattered. The scattering-to-absorption ratio (SAR) is defined as the ratio of scattering power to absorption of radiation by a particle or the ratio of the scattering to the absorption power $[7,8]$. It can be engineered by different parameters such as the size, shape and the surrounding medium. As an example, plasmonic nanoparticles has potential to enhance efficiency of so- lar cells and the plasmonic properties of metal nanoparicles is strongly sensitive to the geometry of nanoparticle [9]. Further, plasmonic nanoparticles can provide a promising platform of potential detection and energy applications due to the enhancement of optical fields and their unprecedented sub-wavelength light focusing capability [10]. Plasmonic nanoparticles exhibit characteristic resonances in absorption and scattering, and they are strong scatterers of light at wavelengths near the plasmon resonance [11]. In addition, plasmonic nanoshells can be utilized as a promising structures to exploit plasmonic resonances for photovoltaic applications because they exhibit controlled optical resonances [12].

There exists a lack of discussion on the SAR of plasmonic nanoparticles with different shapes such as spherical, cylindrical and ellipsoidal types of the nanostructures. In addition, nanorods are nanoparticles elongated into rod-like shapes have received great attention because of their tunable optical properties. In this brief report, however, we investigate the SAR of plasmonic nanoshells of the core-shell type with different shapes because the phenomena of scattering and absorption of light by nanoparticles are central to many science and technology. In fact, control of the SAR in metal nanoparticles helps us to design new plasmonic-base devices with high efficiency and performance which can be used in related applications. However, this work is focused on study of the SAR from different 


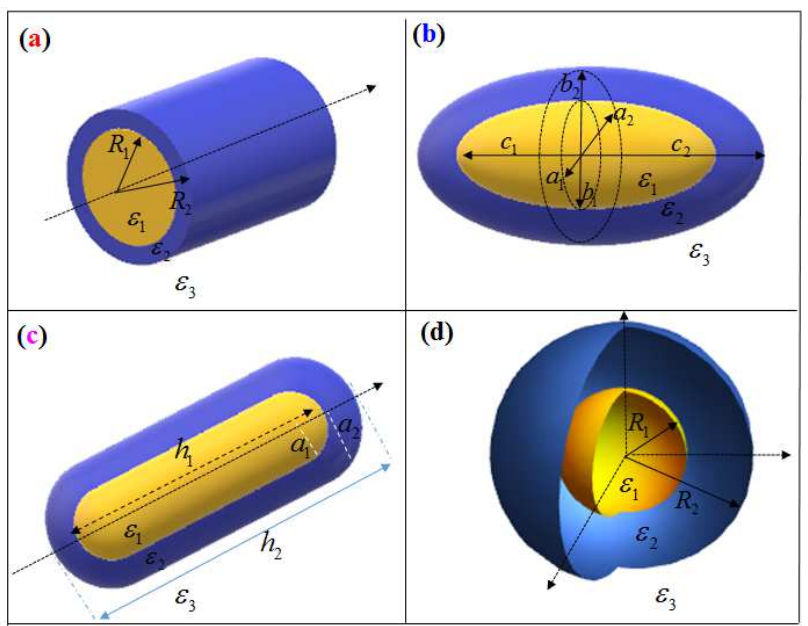

Fig. 1. Schematic diagram of different plasmonic core-shell nanoparticles, (a) cylindrical, b) ellipsoidal, c) nanorod, and d) spherical, embedded in a dielectric environment with permittivity $\varepsilon_{3}$.

plasmonic coated nanoparticles or nanoshells, because the SAR is a criterion to evaluate the relationship between the scattering and absorption cross sections.

\section{ANALYSIS AND DISCUSSION}

The scattering and absorption phenomenon arises from the interaction between the electromagnetic radiation and matter. The light absorption and scattering are determined by the electrostatic polarizability. In order to calculate the optical polarizability of coated nanoparticles, suppose a plasmonic nanoshell depicted in Fig. 1 is illuminated by an electromagnetic wave, in which the size of the proposed nanoparticles is much smaller than the wavelength of incidence light. At the resonance wavelength, metallic nanopaticles strongly interact with the incident light. It is assumed the core and the shell have the respective dielectric constants of $\varepsilon_{1}$ and $\varepsilon_{2}$, and the embedding medium is described by the dielectric function, $\varepsilon_{3}$. We consider only the dipolar response to incident light. So, without going into the full details of dipole polarizability relation of different nanoparticles shown in Fig. 1, the polarizability relation of core-shell nanocylinder and nanosphere geometries are given by, respectively [13-15]

$$
\left\{\begin{aligned}
\alpha^{(\text {cylinder })}(\omega) & =4 \pi \varepsilon_{0} \frac{\left(\varepsilon_{2}-\varepsilon_{3}\right)\left(\left(\varepsilon_{2}-\varepsilon_{1}\right) R_{1}^{2}-\left(\varepsilon_{1}+\varepsilon_{2}\right) R_{2}^{2}\right)+2\left(\varepsilon_{1}-\varepsilon_{2}\right) R_{2}^{2}}{\left(\varepsilon_{1}-\varepsilon_{2}\right)\left(\varepsilon_{2}-\varepsilon_{3}\right) R_{1}^{2}+\left(\varepsilon_{1}+\varepsilon_{2}\right)\left(\varepsilon_{2}+\varepsilon_{3}\right) R_{2}^{2}}, \\
\alpha^{(\text {sphere })}(\omega) & =4 \pi \varepsilon_{0} \frac{\left(\varepsilon_{1}-\varepsilon_{2}\right)\left(\varepsilon_{3}+2 \varepsilon_{2}\right)\left(R_{1} R_{2}\right)^{3}+\left(\varepsilon_{1}+2 \varepsilon_{2}\right)\left(\varepsilon_{2}-\varepsilon_{3}\right) R_{2}^{6}}{2\left(\varepsilon_{1}-\varepsilon_{2}\right)\left(\varepsilon_{2}-\varepsilon_{3}\right) R_{1}^{3}+\left(\varepsilon_{1}+2 \varepsilon_{2}\right)\left(\varepsilon_{2}+2 \varepsilon_{3}\right) R_{2}^{3}},
\end{aligned}\right.
$$

and the polarizability relation of coreshell ellipsoid geometry is given by $[5,13,16-18]$

$$
\alpha^{(\text {ellipsoid })}(\omega)=V \frac{A}{B}
$$

where

$$
\begin{aligned}
A & =\left(\varepsilon_{2}-\varepsilon_{3}\right)\left(\varepsilon_{2}+\left(\varepsilon_{1}-\varepsilon_{2}\right)\left(L_{j}^{(1)}-f L_{j}^{(2)}\right)\right)+f \varepsilon_{2}\left(\varepsilon_{1}-\varepsilon_{2}\right) \\
B & =\left(\varepsilon_{2}+\left(\varepsilon_{1}-\varepsilon_{2}\right)\left(L_{j}^{(1)}-f L_{j}^{(2)}\right)\right)\left(\varepsilon_{3}+\left(\varepsilon_{2}-\varepsilon_{3}\right) L_{j}^{(2)}\right) \\
& +f L_{j}^{(2)} \varepsilon_{2}\left(\varepsilon_{1}-\varepsilon_{2}\right)
\end{aligned}
$$

with

$$
\begin{array}{r}
V=\frac{4}{3} \pi a_{2} b_{2} c_{2}, \quad f=\frac{a_{1} b_{1} c_{1}}{a_{2} b_{2} c_{2}} \\
L_{3}^{(k)}=\frac{1-e_{k}^{2}}{e_{k}^{2}}\left(-1+\frac{1}{2 e_{k}} \ln \frac{1+e_{k}}{1-e_{k}}\right), \\
L_{1}^{(k)}=L_{2}^{(k)}=\frac{1}{2}\left(1-L_{3}^{(k)}, e_{k}=\sqrt{1-\frac{a_{k}^{2}}{c_{k}^{2}} .}\right.
\end{array}
$$

where $j=1,2,3$ denote three principal axes and $k=1,2$. Also, the dipolar polarizability of a coreshell nanorod is given by [5]

$$
\alpha^{(\text {nanorod })}(\omega)=\varepsilon_{3} V_{2} \frac{C}{D}
$$

where

$$
\begin{aligned}
C & =\left(\varepsilon_{2}-\varepsilon_{3}\right)\left(L_{1} \varepsilon_{1}-\left(L_{1}-1\right) \varepsilon_{2}\right) \\
& +\left(V_{1} / V_{2}\right)\left(\varepsilon_{2}-\varepsilon_{1}\right)\left(\left(L_{2}-1\right) \varepsilon_{2}-L_{2} \varepsilon_{3}\right), \\
D & =L_{2}\left(V_{1} / V_{2}\right)\left(L_{2}-1\right)\left(\varepsilon_{2}-\varepsilon_{1}\right)\left(\varepsilon_{2}-\varepsilon_{3}\right) \\
& -\left(\left(L_{1}-1\right) \varepsilon_{2}-L_{1} \varepsilon_{1}\right)\left(L_{2} \varepsilon_{2}-\left(L_{2}-1\right) \varepsilon_{3}\right),
\end{aligned}
$$

with

$$
L_{1}=1 / \sqrt{1+\frac{16 a_{1}^{2}}{\left(4 a_{1}+h_{1}\right)^{2}}}, L_{2}=1 / \sqrt{1+\frac{16 a_{2}^{2}}{\left(4 a_{2}+h_{2}\right)^{2}}},
$$

where, $V_{1}$ and $V_{2}$ are the volume of the core and the shell, respectively.

However, the optical absorption and scattering cross sections are expressed as [19-21]

$$
\left\{\begin{array}{l}
\sigma_{a b s}(\omega) \propto \omega \operatorname{Im}[\alpha(\omega)], \\
\sigma_{\text {scat }}(\omega) \propto \omega^{4}|\alpha(\omega)|^{2},
\end{array}\right.
$$

where the scattering-to-absorption ratio is defined as $S A R=$ $\sigma_{\text {scat }}(\omega) / \sigma_{a b s}(\omega)[7,8]$.

The easy tunability of the optical properties by changing shape of metallic nanoparticles, makes them suitable for a wide range of applications. In order to enhance light-trapping in thin-film optoelectronic devices and increasing the efficiency of them, nanoparticles that exhibit low absorption and large scattering cross sections are good candidate. Owing to the ability of plasmons in metals to confine light on sub-wavelength scales that has been opened up new design possibilities for solar cells [22], in Figs. 2-4, we have investigated the effect of changing the nanoparticle shape on the SAR in plasmonic nanoparticles for different core-shell nanoparticles. For this purpose, the materials chosen are silver $(\mathrm{Ag})$, gold $(\mathrm{Au})$, Bismuth $(\mathrm{Bi})$, silica $\left(\mathrm{SiO}_{2}\right)$ and semiconductor copper chloride $(\mathrm{CuCl})$. The appropriate parameters for $\mathrm{Ag}, \mathrm{Au}, \mathrm{Bi}$ and $\mathrm{CuCl}$ can be found in tables 1 and 2 , respectively. Also, table 3 shows radii of different plasmonic coated nanoparticles depicted in Fig. 1. In addition, the surrounding medium of nanoparticle is silicon having a refractive index of 2.25.

It can be seen the SAR is strongly influenced by the changing of the nanoparticle shape as shown in Figs. 2-4. The low-loss noble metals Ag and Au can be used for plasmonics in the visible range and NIR, and the poor metal bismuth can be used for 
Table 1. Drude model parameters for Bismuth (Bi) [23], silver $(\mathrm{Ag})$ and gold $(\mathrm{Au})[24]$.

\begin{tabular}{|c|c|c|c|}
\hline & $\varepsilon_{\infty}$ & $\omega_{p}(\mathrm{eV})$ & $\gamma(\mathrm{eV})$ \\
\hline $\mathrm{Au}$ & 6.9 & 8.9 & 0.07 \\
\hline $\mathrm{Ag}$ & 3.57 & 9.2 & 0.052 \\
\hline $\mathrm{Bi}$ & 3.702 & 9.004 & 0.878 \\
\hline
\end{tabular}

Table 2. Lorentz model parameters for $\mathrm{CuCl}[25,26]$.

\begin{tabular}{|c|c|c|c|c|}
\hline & $\varepsilon_{\infty}$ & $\omega_{0}(\mathrm{eV})$ & $\gamma(\mu \mathrm{eV})$ & A(oscillator strength) \\
\hline $\mathrm{CuCl}$ & 5.59 & 3.3022 & 49.206 & 632 \\
\hline
\end{tabular}

Table 3. The core and shell radius of different nanoshells depicted in Fig. 1.

\begin{tabular}{|c|c|}
\hline & Core and shell radius \\
\hline Fig. 1a & {$\left[\mathrm{R}_{1}, R_{2}\right]=[5,10] \mathrm{nm}$} \\
\hline Fig. 1b & {$\left[\mathrm{a}_{1}, b_{1}, c_{1}\right]=[2,3,5] \mathrm{nm} \quad \& \quad\left[a_{2}, b_{2}, c_{2}\right]=[7,8,10] \mathrm{nm}$} \\
\hline Fig. 1c & {$\left[\mathrm{a}_{1}, a_{2}\right]=[5,10] \mathrm{nm} \quad \& \quad\left[h_{1}, h_{2}\right]=[5,8] \mathrm{nm}$} \\
\hline Fig. 1d & {$\left[\mathrm{R}_{1}, R_{2}\right]=[5,10] \mathrm{nm}$} \\
\hline
\end{tabular}
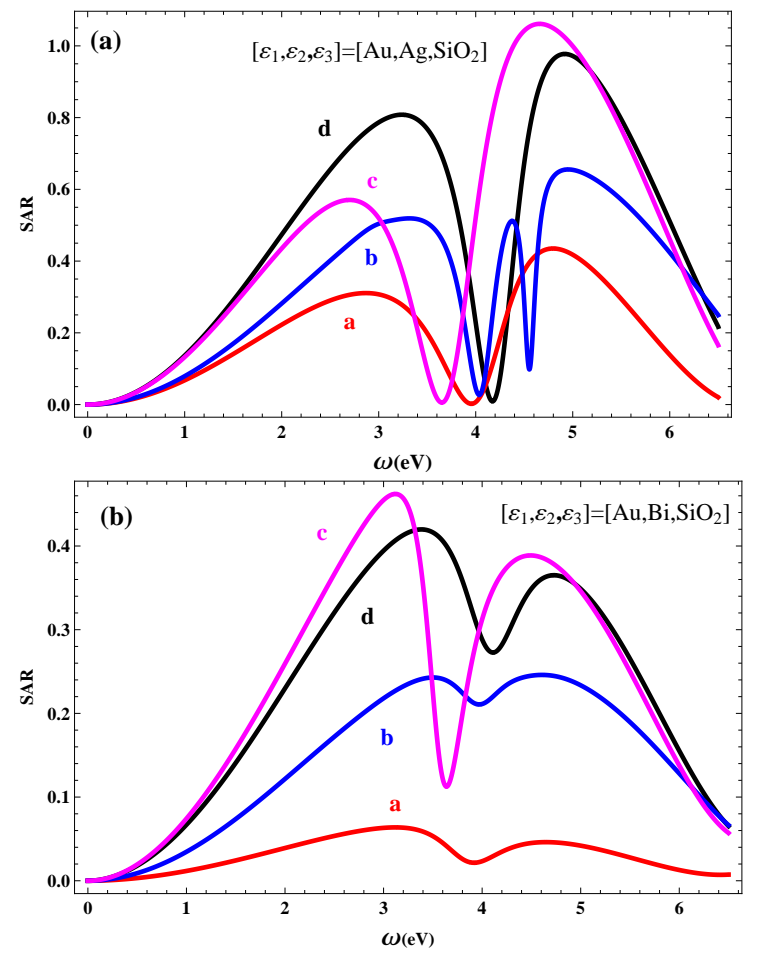

Fig. 2. Comparison between calculated spectra of the SAR for different plasmonic nanoshells. The nanoshell is composed of a gold core and a concentric shell of Ag and Bi for (a) and (b) plots, respectively. plasmonics in the near to far ultraviolet range [23]. The comparison of SAR between Au core/Ag shell and Au core/Bi shell nanoprticles is shown in Fig. 2. In Fig. 2a the coating layer is $\mathrm{Ag}$, in which in Fig. $2 \mathrm{~b}$ the coating layer is Bi. Both the intensity and position of the SAR are sensitive to the nanoparticle shape. As demonstrated in this figure, the intensity of SAR is weak when the shell is Bi. This means that the absorption intensity by a Au core/Bi shell structure is stronger than the scattering intensity, in which for photovoltaic applications it is particularly important to minimize absorption.
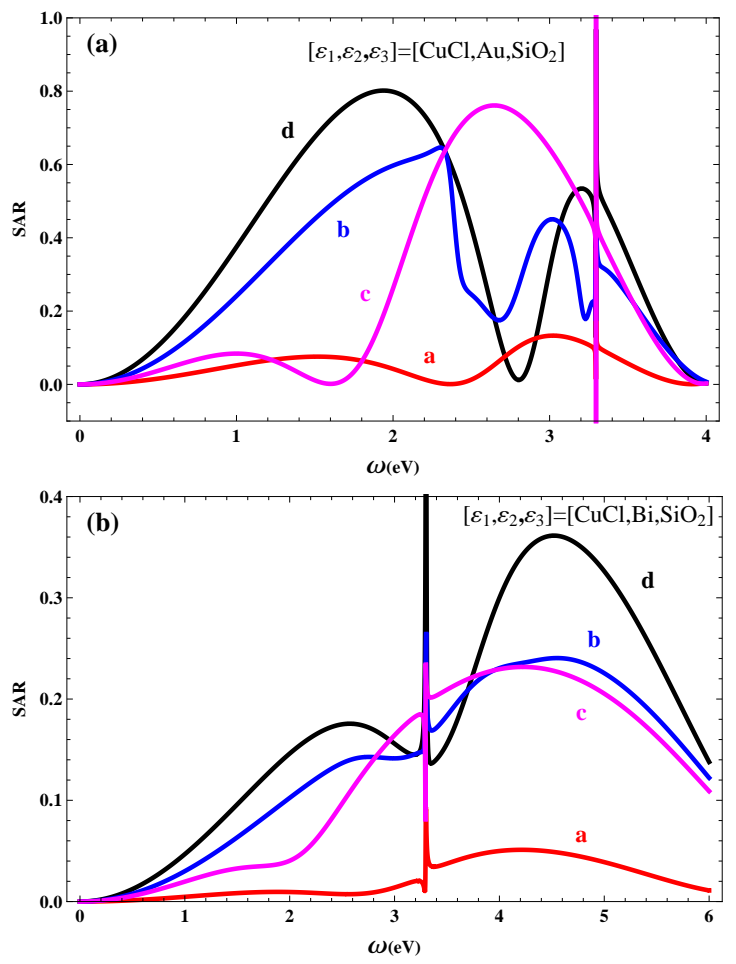

Fig. 3. Comparison between calculated spectra of SAR for different plasmonic nanoshells. The nanoshell is composed of a $\mathrm{CuCl}$ core and a concentric shell of $\mathrm{Au}$ and $\mathrm{Bi}$ for (a) and (b) plots, respectively.

Combining metal and semiconductor nanoparticles in one system leading to plasmon-exciton coupling (also called plexciton) which has attracted a lot of interest recently due to a wide range of their potential applications as biosensors and solar cells [27]. To investigate the SAR in a metal-semiconductor nanostructure, we have considered a plexcitonic nanoshell consists of a semiconductor $\mathrm{CuCl}$ core that exhibits a strong exciton resonance and a very small spectral width [28], coated by a metallic shell which can strongly absorb and scatter light due to its ability to support surface plasmon resonances as demonstrated in Fig. 3. It is interesting to note that the properties of both the plasmon and the exciton can be modified by strong coupling between the localized surface plasmon resonances in metallic nanostructures and the local excitons in semiconductors [29]. As observed in Fig. 3, a sharp peak appears in the SAR spectrum at $3.3 \mathrm{eV}$ when the core is $\mathrm{CuCl}$ and the shell is gold, while a very sharp and strong peak appears at about $3.2 \mathrm{eV}$ when the core is $\mathrm{CuCl}$ and the shell is bismuth. In fact, the appearance of sharp peak is due to the exciton resonance of semiconductor $\mathrm{CuCl}$ core.

For enhancement of light-trapping in silicon solar cells, we 

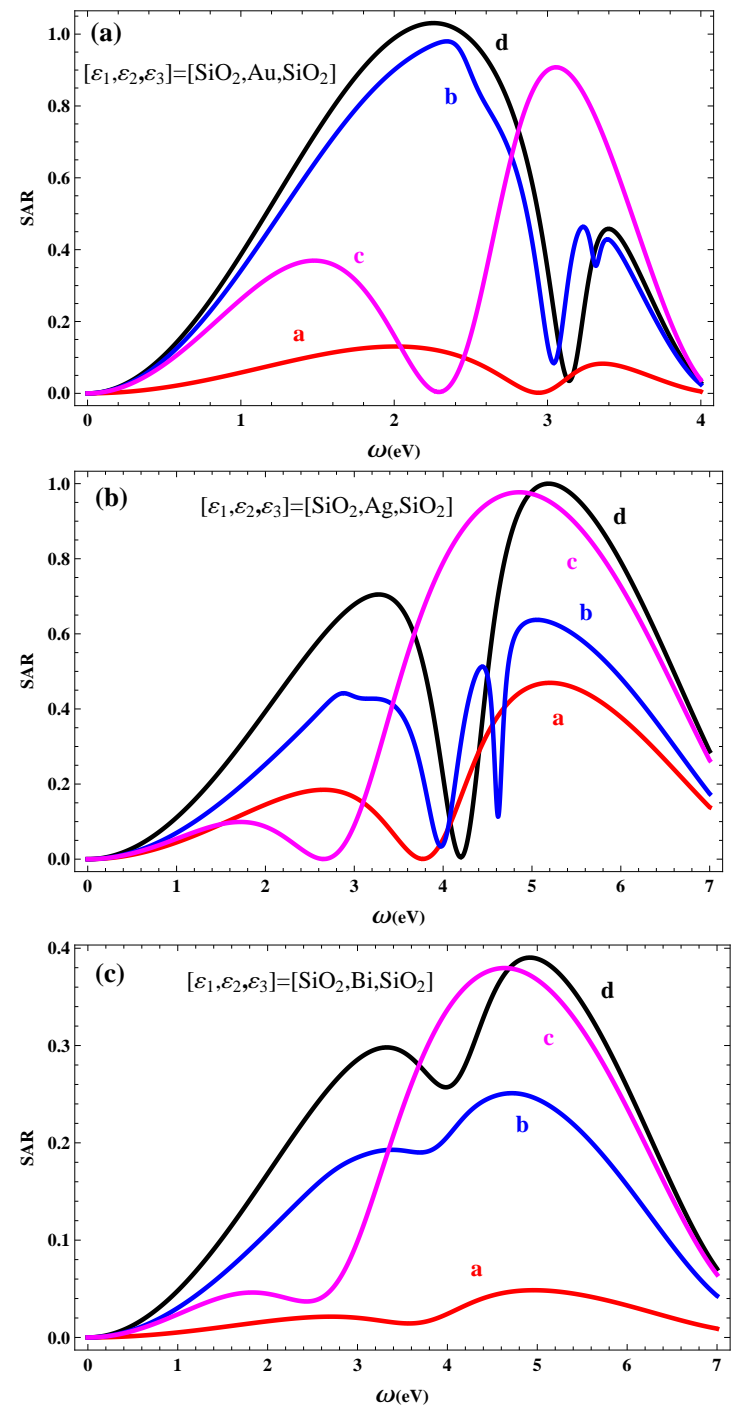

Fig. 4. Comparison between calculated spectra of SAR for different plasmonic nanoshells. The nanoshell is composed of a silica core $\left(\mathrm{SiO}_{2}\right)$ and a concentric shell of $\mathrm{Au}, \mathrm{Ag}$ and $\mathrm{Bi}$ for (a), (b) and (c) plots, respectively. need nanoparticles that exhibit low absorption in the visible and NIR, and large scattering cross-sections across the useful solar spectrum [30]. On this basis, the SAR spectra for different silicon-based nanoparticles is potted for $\mathrm{SiO}_{2}$ core/Au shell, $\mathrm{SiO}_{2}$ core/Ag shell and $\mathrm{SiO}_{2}$ core/Bi shell nanoparticles as shown in Fig. 4. The results show that the SAR decreases when $\mathrm{SiO}_{2}$ coated with a poor metal as $\mathrm{Bi}$. In addition, the cross-sectional shape of nanoparticle is seen to affect the peak position and width of the spectrum that is important for photovoltaic applications. Since, the coating shell increases the light scattering, coating $\mathrm{SiO}_{2}$ with a plasmonic shell such as silver or gold can increase the SAR.

\section{CONCLUSION}

In summary, a comparative study of the scattering-toabsorption ratio in different nanoparticles such as nanosphere, nanorod, nanoellipsoid and nanocylider with core-shell geometry in a large spectral range has been investigated which makes them suitable for a wide range of applications. It is found that the SAR is strongly influenced by the changing of the nanoparticle shape and all surface plasmon resonance peaks are tunable by changing the shape of particle. Our results give helpful insights in the development of photovoltaic cells and sensors.

\section{REFERENCES}

1. A. Agrawal, I. Kriegel, and D. Milliron, J. Phys. Chem. C 119, 6227 (2015).

2. P. Latimer, Ann. Rev. Biophys. Bioeng. 11, 129 (1982).

3. P. K. Jain, K. S. Lee, I. H. El-Sayed, and M. A. El-Sayed, J. Phys. Chem. B 110, 7238 (2006).

4. X. Fan, W. Zheng and D. J. Singh, Light: Science \& Applications 3, e179 (2014).

5. R. lovine, L. La Spada, and L. Vegni, IEEE Trans. Magnetics. 50, 7004004 (2014).

6. S. Marhaba, Noble and Precious Metals: Properties, Nanoscale Effects and Applications, eds. M. Seehra and A. Bristow (IntechOpen, London, 2018), p. 43.

7. S-W. Baek, J. Noh, C-H. Lee, B. S. Kim, M-K. Seo and J-Y. Lee, Sci. Reports 3, 1726 (2013).

8. P. Yu, Y.Yao, J. Wu, X. Niu, A. L. Rogach and Z. Wang, Sci. Rep. 7, 7696 (2017).

9. M. W. Knight and N. J. Halas, New J. Phys. 10, 105006 (2008).

10. S. V. Boriskina, H. Ghasemi and G. Chen, Materials Today 16, 375386 (2013).

11. K.R. Catchpole, and A. Polman, Opt. Express 16, 21793 (2008).

12. W. Raja, A. Bozzola, P. Zilio, E. Miele, S. Panaro, H. Wang, A. Toma, A. Alabastri, F. De Angelis and R. P. Zaccaria, Sci. Rep. 6, 24539 (2016).

13. C.F. Bohren, D.R. Huffman, Absorption and Scattering of Light by Small Particles (Wiley, New York, 1983)

14. J. Zhu, Physica E 27, 296 (2005).

15. N. Daneshfar and K. Bazyari, Appl. Phys. A 116, 611 (2014).

16. M. Liu and P. Guyot-Sionnest, J. Phys. Chem. B 108, 5882 (2004).

17. M. Liu and P. Guyot-Sionnest, J. Mater. Chem. 16, 3942 (2006).

18. J. Zhu, J. Nanoscience and Nanotechnology 7, 1059 (2007).

19. W. Ni, T. Ambjornsson, S. P. Apell, H. Chen, and J. Wang, Nano Lett. 10, 77 (2010).

20. D.Gulen, J. Phys. Chem. B 117, 11220 (2013).

21. L. Escoubas, M. Carlberg, J. Le Rouzo, F. Pourcin, J. Ackermann, O. Margeat, C. Reynaud, D. Duche, J-J Simon, R-M. Sauvagec, G. Berginc, Prog. Quantum Electronics 63, 1-20 (2019) .

22. M. A. Green and S. Pillai, Nat. Photonics 6130 (2012).

23. J. M. McMahon, G. C. Schatza and S. K. Gray, Phys. Chem. Chem. Phys. 15, 5415 (2013).

24. P. R. West, S. Ishii, G. V. Naik, N. K. Emani, V. M. Shalaev, and A. Boltasseva, Laser Photonics Rev. 4, 795 (2010). 
25. J. T. Manassah, Phys. Rev A 87, 053845 (2013) .

26. S. Jiang, Q. Y. Xie, D. Wu, Appl. Phys. B 119, 355 (2015).

27. V. V. Savchuk, R. V. Gamernyk, I. S. Virt, S. Z. Malynych , and A. O. Pinchuk, AIP Advances 9, 045021 (2019).

28. V. Yannopapas and N. V. Vitanov, Phys. Rev. B 74, 193304 (2006).

29. D. Wu, Y. Cheng, X-W. Wu, and X-J. Liu, J. Opt. Soc. Am. B 31, 2273 (2014).

30. T. L. Temple and D. M. Bagnall, J. Appl. Phys 109, 084343 (2011). 


\section{Figures}

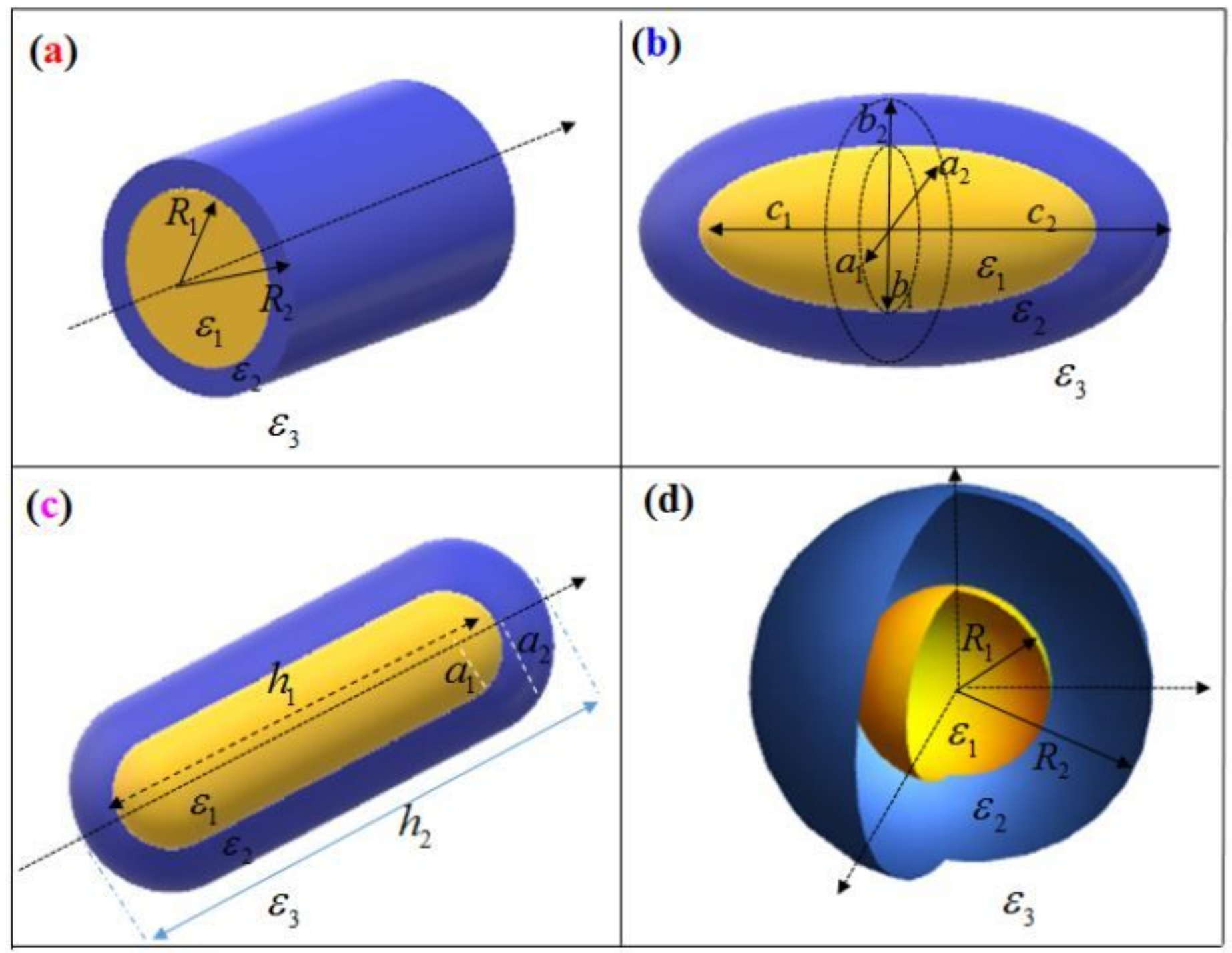

Figure 1

Schematic diagram of different plasmonic core-shell nanoparticles, (a) cylindrical, b) ellipsoidal, c) nanorod, and d) spherical, embedded in a dielectric environment with permittivity $\varepsilon 3$. 

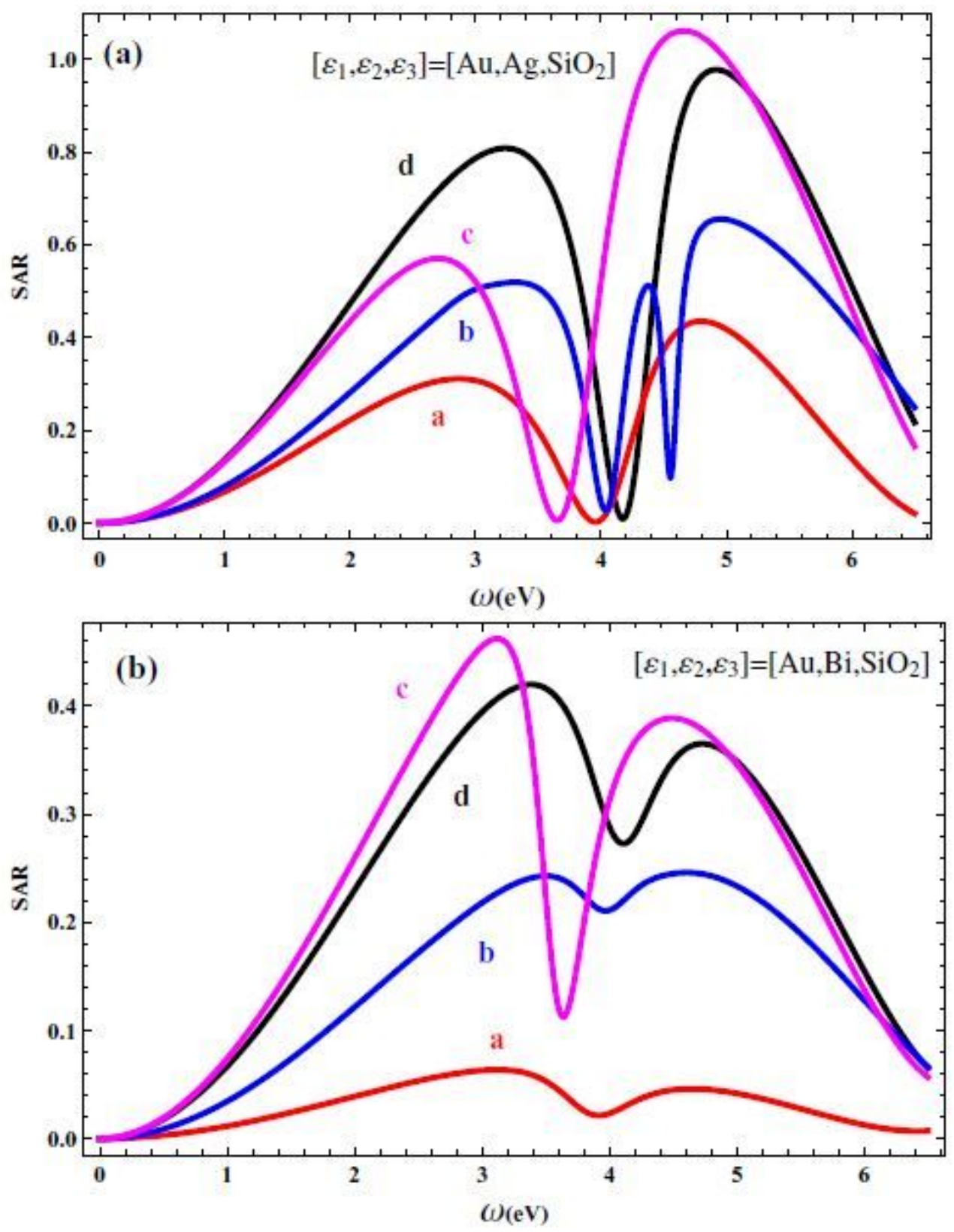

Figure 2

Comparison between calculated spectra of the SAR for different plasmonic nanoshells. The nanoshell is composed of a gold core and a concentric shell of Ag and Bi for (a) and (b) plots, respectively. 

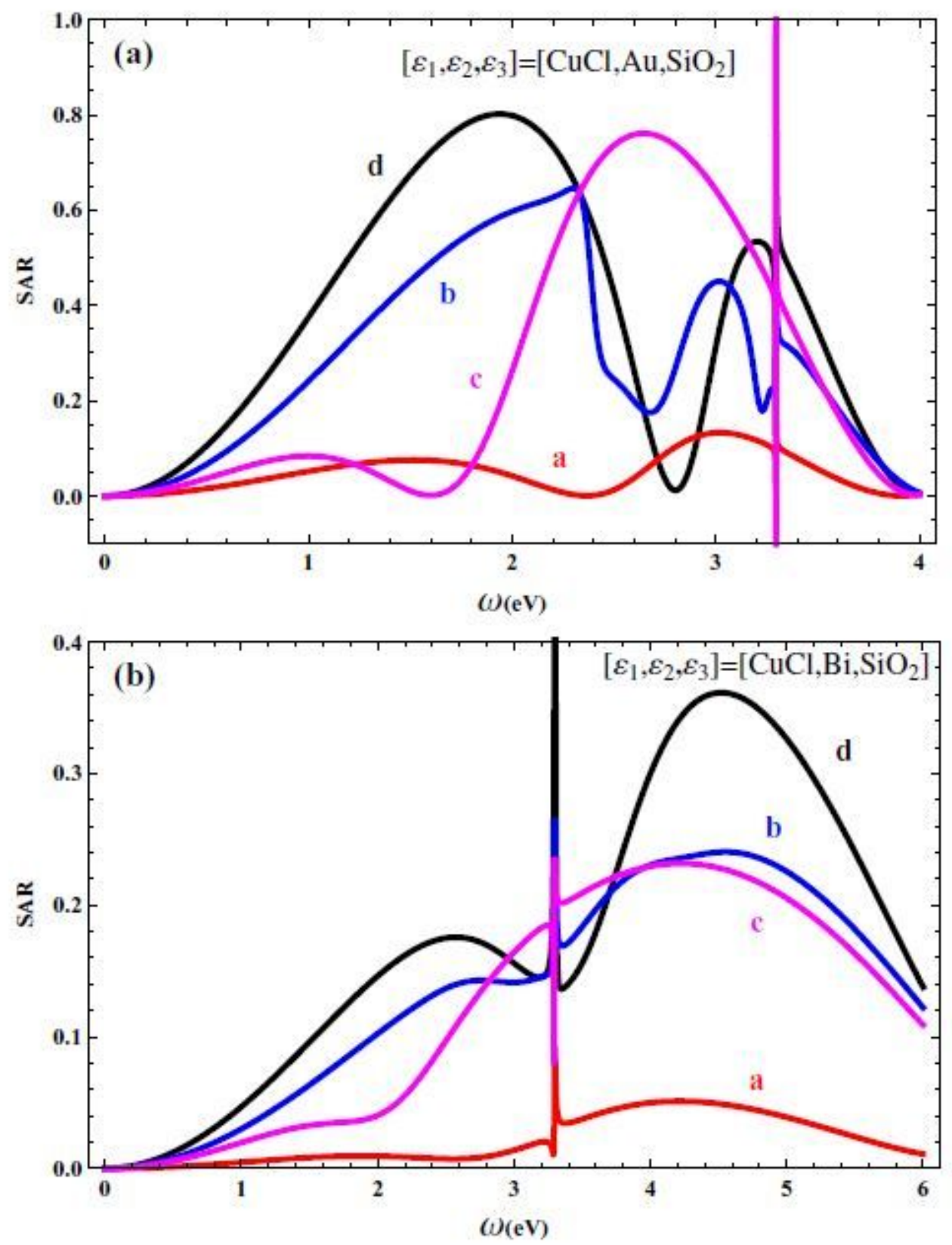

Figure 3

Comparison between calculated spectra of SAR for different plasmonic nanoshells. The nanoshell is composed of a CuCl core and a concentric shell of Au and Bi for (a) and (b) plots, respectively. 

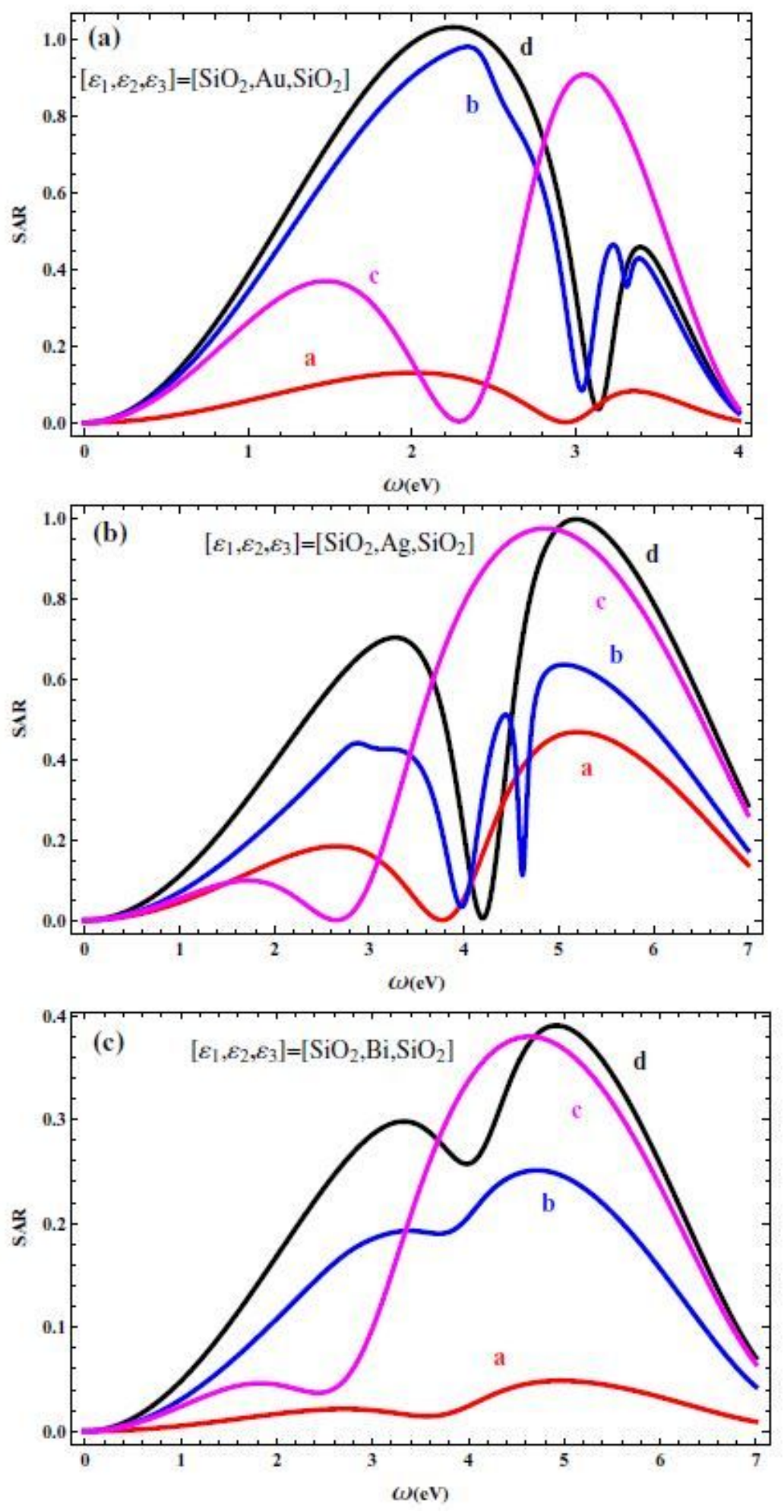

Figure 4

Comparison between calculated spectra of SAR for different plasmonic nanoshells. The nanoshell is composed of a silica core (SiO2) and a concentric shell of Au, Ag and Bi for (a), (b) and (c) plots, respectively. 